Meta

Journal des traducteurs

Translators' Journal

\title{
La coordination des adjectifs modificateurs en russe et en français : la conjonction russe I et la conjonction française ET
}

\section{Lidija Iordanskaja et Igor Mel’čuk}

Volume 55, numéro 1, mars 2010

Le parcours du sens : d'une langue à l'autre — Mélanges offerts à André Clas

The Way of Meaning: From a Language to Another - Collection of Articles Offered to André Clas

URI : https://id.erudit.org/iderudit/039611ar

DOI : https://doi.org/10.7202/039611ar

Aller au sommaire du numéro

\section{Éditeur(s)}

Les Presses de l'Université de Montréal

\section{ISSN}

0026-0452 (imprimé)

1492-1421 (numérique)

Découvrir la revue

Citer cet article

Iordanskaja, L. \& Mel'čuk, I. (2010). La coordination des adjectifs modificateurs en russe et en français : la conjonction russe I et la conjonction française ET. Meta, 55(1), 168-186. https://doi.org/10.7202/039611ar
Résumé de l'article

Le présent article établit les régularités d'emploi de coordination, par opposition à la codépendance, de deux adjectifs modifiant un nom en français et en russe. La possibilité de coordination par la conjonction française ET / russe I est basée sur l'homogénéité des adjectifs (un regard lourd et morose), tandis que celle de leur codépendance se base sur leur hétérogénéité (une voiture rouge fiable). On distingue l'homogénéité sémantique (induite par le sens lexicographique des adjectifs) et l'homogénéité pragmatique (imposée par le locuteur voulant souligner la similitude situationnelle de deux adjectifs sémantiquement hétérogènes). Pour caractériser l'homogénéité des adjectifs, l'article propose une classification sémantique. Une comparaison systématique de la coordination et de la codépendance adjectivales dans les deux langues démontre que, dans la majorité des cas, le français préfère la coordination des adjectifs et le russe la codépendance : par exemple, un ciel haut et bleu $<{ }^{*}$ un ciel hautbleu $>\sim$ vysokoe sinee nebo $<{ }^{*}$ vysokoe i sinee nebo $>$. 


\title{
SYNTAXE ET SÉMANTIQUE
}

\section{La coordination des adjectifs modificateurs en russe et en français : la conjonction russe I et la conjonction française ET}

\author{
LIDIJA IORDANSKAJA* \\ Université de Montréal, Montréal, Canada \\ lidija.iordanskaja@umontreal.ca \\ IGOR MEL'ČUK ${ }^{*}$ \\ Université de Montréal, Montréal, Canada \\ igor.melcuk@umontreal.ca
}

\section{RÉSUMÉ}

Le présent article établit les régularités d'emploi de coordination, par opposition à la codépendance, de deux adjectifs modifiant un nom en français et en russe. La possibilité de coordination par la conjonction française ET / russe I est basée sur l'homogénéité des adjectifs (un regard lourd et morose), tandis que celle de leur codépendance se base sur leur hétérogénéité (une voiture rouge fiable). On distingue l'homogénéité sémantique (induite par le sens lexicographique des adjectifs) et l'homogénéité pragmatique (imposée par le locuteur voulant souligner la similitude situationnelle de deux adjectifs sémantiquement hétérogènes). Pour caractériser l'homogénéité des adjectifs, l'article propose une classification sémantique. Une comparaison systématique de la coordination et de la codépendance adjectivales dans les deux langues démontre que, dans la majorité des cas, le français préfère la coordination des adjectifs et le russe la codépendance: par exemple, un ciel haut et bleu <*un ciel haut bleu> $\sim$ vysokoe sinee nebo <"vysokoe i sinee nebo>.

\section{ABSTRACT}

The present paper establishes regularities in the use of coordination vs. co-dependence of two adjectives that modify a noun in French and Russian. The possibility of coordination by the conjunction Fr. ET 'and'/Rus. I 'and' is based on homogeneity of adjectives, while that of their co-dependence on their heterogeneity. Two types of adjective homogeneity are distinguished: semantic homogeneity (induced by the lexicographic meaning of adjectives) and pragmatic homogeneity (determined by the speaker who wants to emphasize the situational similarity of two adjectives that are semantically heterogeneous). In order to characterize the homogeneity of adjectives, the paper proposes their semantic classification. A systematic comparison of adjectival coordination with codependence in both languages shows that in a majority of cases French prefers coordination of adjectives, while Russian prefers their co-dependence: for instance, 'a high blue sky' is rendered in French as un ciel haut et bleu <*un ciel haut bleu>, and in Russian as vysokoe sinee nebo <*vysokoe i sinee nebo>.

\section{MOTS-CLÉS/KEYWORDS}

français, russe, conjonction ET/I, coordination/codépendance des adjectifs modificateurs

French, Russian, ET/I conjunction, coordination/co-dependence of modifying adjectives 


\section{Problème posé}

Dans une biographie de Lou Andreas-Salomé, figure très connue de l'univers culturel européen du début du $\mathrm{Xx}^{\mathrm{e}}$ siècle ${ }^{1}$, on trouve une photo de Friedrich Nietzsche, qui était follement épris d'elle, avec la légende suivante, qui n'a rien pour surprendre un francophone:

(1) a. Nietzsche. Génial et amoureux.

Ceci, cependant, est choquant pour un Russe; la traduction littérale de (1a) serait inacceptable et même ridicule:

(1) b. Nicše. *Genial'nyj i vljublënnyj².

Le russe ne permet pas de coordonner par la conjonction I ('et') deux adjectifs modificateurs dont l'un décrit une caractéristique permanente de quelqu'un (génial, beau, grand...) et l'autre, un état temporaire (amoureux, malade, fatigué...). La bonne façon de traduire (1)a sans changer trop drastiquement la structure serait de dire (1)c:

(1) c. Nič̌e. Vljublënnyj genij.

'Nietzsche. Génie amoureux'

À noter que, si au lieu d'un nom propre, Nietzsche, on avait un nom commun, filosof 'philosophe' par exemple, on pourrait utiliser en russe la construction avec deux adjectifs modificateurs sans conjonction (1)d:

(1) d. vljublënnyj genial'nyj filosof

'[le] philosophe génial amoureux'

C'est donc la présence de la conjonction I ('et') qui rend le syntagme (1)b inacceptable. À cela on peut ajouter un autre type d'exemple où ET n'admet pas la traduction par I:

(2) a. une mondialisation plus juste et qui profite à tous

b. ${ }^{\star}$ globalizacija, bolee spravedlivaja $\boldsymbol{i}$ kotoraja vygodna dlja vsex

L'exemple (2)b illustre un phénomène général, typique du russe: contrairement au français, le russe interdit la coordination d'un adjectif avec une relative. Le syntagme (2)a peut être traduit, par exemple, comme suit:

(2) c. (i) globalizacija, bolee spravedlivaja i vygodnaja dlja vsex 'mondialisation plus juste et profitable à tout.le.monde'

(ii) globalizacija, kotoraja javljaetsja bolee spravedlivoj i vygodnoj dlja vsex 'mondialisation qui est plus juste et profitable à tout.le.monde's

Les exemples (1) et (2) montrent qu'au moins dans les contextes considérés, la conjonction russe I est d'un emploi plus restreint que la conjonction française ET. Pour mieux formuler les restrictions correspondantes en français et en russe, nous nous limiterons à un seul lexème des conjonctions considérées: une acception qui représente le sens de la conjonction logique «pure» $[=\&$ ou $\wedge]$. Nous allons considérer 
ce lexème dans une seule construction syntaxique, à savoir entre deux adjectifs coordonnés utilisés en tant que modificateurs d'un nom. Cette construction correspond à la situation où le locuteur attribue à une entité deux caractéristiques, ces caractéristiques formant un ensemble conjonctif, c'est-à-dire 'ensemble ${ }_{\mathrm{et}}$ ' (voir plus bas) ${ }^{4}$.

Nous excluons de notre analyse:

- Les constructions où la chaîne d'adjectifs coordonnés contient plus de deux adjectifs;

- Les constructions où la paire d'adjectifs est un complément attributif d'une copule, car les règles d'emploi de I dans cette construction sont moins strictes; ainsi, au tout début d'un texte, on observe le contraste en (3):

(3) a. ${ }^{*}$ Na stole ležala bol'šaja $\boldsymbol{i}$ žëltaja roza.

'sur la table était une grande et jaune rose' <correct: Na stole ležala bol'šaja žëltaja roza> 'sur la table était une grande jaune rose'

b. Na stole ležala roza; roza byla bol'šaja $\boldsymbol{i}$ žëltaja. 'sur la table était une rose; la rose était grande et jaune'

- Les constructions où la paire d'adjectifs modifie un nom au pluriel, car de telles expressions peuvent être ambiguës. Ainsi, le syntagme

(4) a. des drapeaux rouges et blancs

signifie soit un ensemble de drapeaux dont chacun est rouge et blanc, soit un ensemble de drapeaux rouges et de drapeaux blancs. Même quand les deux adjectifs coordonnés n'entraînent pas d'ambiguïté, il ne s'agit pas de deux caractéristiques simultanées d'une même entité: (4)b parle de quelques livres français et de quelques livres allemands:

(4) b. des livres français et allemands

Cependant, nous admettons dans nos données quelques noms pluriels s'ils dénotent une seule entité (yeux, dents, doigts [d'une personne] ou [marches d'un escalier]).

- Les constructions où l'un des deux adjectifs forme avec le nom modifié une locution; dans ce cas, la coordination des adjectifs est impossible:

(5) *quartier général et temporaire *corps expéditionnaire et motorisé

- Les constructions où le deuxième adjectif désigne une sous-classe d'entités désignées par le premier:

(6) église chrétienne évangéliste église chrétienne et évangéliste

Ici, la caractéristique 'évangéliste' réfère à une sous-classe d’entités 'chrétiennes'.

Comme il a été dit, par comparaison avec le français, le russe possède au moins deux types de contraintes plus restrictives sur l'emploi de la conjonction I dans les constructions traitées dans cet article: des contraintes sémantiques et des contraintes syntaxiques. Ces dernières sont assez évidentes; nous ne nous intéresserons donc ici qu'aux contraintes sémantiques. 


\section{Contraintes sémantiques sur la coordination des adjectifs modificateurs}

En comparant la construction russe où deux adjectifs modifient un nom avec sa traduction française ou vice versa, on note immédiatement qu'il est très souvent possible ou même nécessaire d'utiliser ET en français, tandis qu'en russe, l'apparition de la conjonction I - dans un contexte neutre - est inacceptable:

(7) a. un sentier étroit et sablonneux

$$
\begin{aligned}
& \sim \text { uzkaja }\left({ }^{*} i\right) \text { pesčanaja dorožka } \\
& \sim \text { umnoe }\left({ }^{*}\right. \text { i) krugloe lico } \\
& \sim \text { neprijtanyj }\left({ }^{*}\right. \text { i) tjažëlyj vzgljad }
\end{aligned}
$$

b. un visage rond et intelligent

c. un regard lourd et désagréable

d. le pauvre et ardent cadet

$\sim$ bednyj $\left({ }^{*}\right.$ i) strastnyj junker

Cela ne veut pas dire que le français admet n'importe quelles coordinations de deux adjectifs par ET:

(8) a. *une vallée triangulaire et profonde

b. *une voiture rouge et fiable

c. ${ }^{*}$ un sommet conique et enneigé

d. *une église blanche et miniature une vallée triangulaire profonde / une profonde vallée triangulaire

$\sim$ une voiture rouge fiable

un sommet conique enneigé

une église blanche miniature

Il faut donc formuler les contraintes en spécifiant pour chaque langue l'emploi de ET / I entre deux adjectifs modificateurs. Mais, avant de le faire, nous nous devons de présenter notre description du phénomène en question de façon générale sur les plans sémantique et syntaxique.

Pour fixer les idées, considérons un sémantème nominal ' $\sigma$ ' dont le référent reçoit en même temps deux caractéristiques, ' $\sigma_{1}^{\prime}$ ' et ' $\sigma_{2}$ ', c'est-à-dire que ' $\sigma$ ' doit être un argument de ces deux prédicats. Dans ce cas, nous avons, à la surface, trois constructions syntaxiques porteuses de sens légèrement différents (' $\sigma$ ' est exprimée par $N$, ' $\sigma_{1}$ ' et ' $\sigma_{2}$ ' - par $\operatorname{Adj}_{1}$ et $\mathrm{Adj}_{2}$; tableau 1).

\begin{tabular}{|c|c|}
\hline 1. $\underset{\text { ATtR }}{\mathrm{N}} \rightarrow \mathrm{Adj}_{1} \mathrm{Adj}_{2}$ & $\begin{array}{l}\text { tëplyj tixij večer } \\
\text { 'douce calme soirée' } \\
\text { une église blanche miniature }\end{array}$ \\
\hline 2. $\mathrm{N}$ ATTR $\rightarrow \operatorname{Adj}_{1} \operatorname{COORD} \rightarrow \mathrm{I} / \mathrm{ET} \rightarrow \operatorname{Adj}_{2}$ & $\begin{array}{l}\text { tëplyj i tixij večer } \\
\text { 'douce et calme soirée' } \\
\text { une soirée douce et calme }\end{array}$ \\
\hline 3. $\mathrm{N}$ ATTR $\rightarrow \mathrm{Adj}_{1}$ QUASI-COORD $\rightarrow \mathrm{I} / \mathrm{ET} \rightarrow \mathrm{Adj}_{2}$ & $\begin{array}{l}\text { sil'noe, vydelitel'noe udarenie } \\
\text { 'fort, délimitatif accent' } \\
\text { un accent fort, délimitatif }\end{array}$ \\
\hline
\end{tabular}

TABLeAu 1

Constructions avec deux adjectifs modificateurs en russe et en français

La première construction, qui représente la subordination en parallèle, ou la codépendance, des adjectifs modificateurs, correspond à deux caractéristiques indépendantes. La deuxième construction, la coordination des adjectifs modificateurs, signale la réunion des caractéristiques en question dans un ensemble. Et dans la troisième, avec la quasi-coordination des adjectifs (une pause entre les deux, et les contours tombants sur chacun: $\searrow \mid \searrow$ ), le deuxième adjectif constitue un ajout informationnel au premier (une explication, une précision, un rehaussement) ${ }^{5}$. En conformité avec 
cela, nous proposons que les caractéristiques ' $\sigma_{1}$ ' et ' $\sigma_{2}$ ' soient représentées dans la Structure Sémantique [= SSém] du type Sens-Texte de l'une des trois façons suivantes $^{6}$ (tableau 2).

TABLEAU 2

Configurations de sémantèmes représentant les constructions avec deux adjectifs modificateurs

\begin{tabular}{|l|l|l|}
\hline $\begin{array}{l}\text { A. ' } \sigma_{1}^{\prime} \text { et ' } \sigma_{2}^{\prime} \text { apparaissent } \\
\text { comme indépendantes }\end{array}$ & $\begin{array}{l}\text { B. ' } \sigma_{1}^{\prime} \text { et ' } \sigma_{2}^{\prime} \text { apparaissent comme } \\
\text { formant un ensemble conjonctif }\end{array}$ & $\begin{array}{l}\text { C. ' } \sigma_{2}^{\prime} \text { apparaît comme un ajout } \\
\text { à } \sigma_{1}^{\prime}\end{array}$ \\
\hline
\end{tabular}

Pour simplifier notre exposé, nous n'examinerons que les deux premiers cas, $\mathbf{A}$ et $\mathbf{B}$. La configuration $\mathbf{A}$ présuppose que les caractéristiques ' $\sigma_{1}$ ' et ' $\sigma_{2}$ ' sont assez hétérogènes, de sorte qu'il est naturel pour le locuteur de les présenter comme indépendantes. Par contre, dans la configuration $\mathbf{B}$, les caractéristiques ' $\sigma_{1}$ ' et ' $\sigma_{2}$ ' sont réunies selon un aspect commun, c'est-à-dire que ' $\sigma_{1}$ ' et ' $\sigma_{2}$ ' sont présentées comme plutôt homogènes. En disant cela, nous suivons l'analyse de la coordination linguistique par plusieurs linguistes slaves (Peškovskij 1934: 392; Wierzbicka 1980 : 223-285, spécialement 251-254 - les paragraphes sur un «dénominateur commun», nécessaire dans le sémantisme des éléments coordonnés; Sannikov 1989: 188; Boguslavskij 1996: 397)

Donc, selon la nature de ' $\sigma_{1}$ ' et ' $\sigma_{2}$ ', chacune des configurations $\mathbf{A}$ et $\mathbf{B}$ peut être correcte ou incorrecte:

- Soit ' $\sigma_{1}$ ' et ' $\sigma_{2}$ ' sont trop homogènes pour ne pas être réunies dans un ensemble; la configuration $\mathbf{A}$ est à rejeter.

- Soit ' $\sigma_{1}$ ' et ' $\sigma_{2}$ ' sont trop hétérogènes pour être réunies dans un ensemble; la configuration $\mathbf{B}$ est à rejeter.

- Soit, enfin, $\mathbf{A}$ et $\mathbf{B}$ sont toutes les deux correctes: ' $\sigma_{1}$ ' et ' $\sigma_{2}$ ' ne sont ni trop homogènes ni trop hétérogènes, ce qui donne une marge de manœuvre au locuteur; les deux structures sémantiques légèrement différentes sont possibles.

Soulignons que la différence sémantique que nous essayons d'expliciter est très ténue: elle ne correspond qu'à l'intention du locuteur et ne reflète aucune différence dans la situation extralinguistique. Dans la traduction entre le français et le russe, on est souvent forcé de la négliger, sans changer l'information pertinente. Ainsi, le syntagme des yeux noirs et brillants (au-dessus de 100000 occurrences sur l'Internet), où le locuteur exprime une perception unie de ces deux caractéristiques, décrit les mêmes yeux que le syntagme des yeux noirs brillants (47000 occurrences), sans une telle perception. Le caractère subjectif de cette distinction est cause de pas mal de désaccords et d'hésitations chez les informateurs qui jugeaient de l'acceptabilité de nos exemples hors contexte. Souvent, quand des options sont possibles, il s'agit plutôt 
de préférences (plus ou moins fortes) que de règles absolues; dans de tels cas, nos astérisques sont à interpréter cum grano salis.

La correction de la SSém de départ $\mathbf{A}$ et $\mathbf{B}$ se laisse décrire de la façon suivante. Une configuration de sémantèmes de la forme $\mathbf{A}$ ou $\mathbf{B}$ doit être soumise aux règles de correction qui vérifient l'absence de l'homogénéité des caractéristiques ' $\sigma_{1}$ ' et ' $\sigma_{2}$ ' (pour A) ou leur homogénéité (pour B). L'homogénéité des caractéristiques est déterminée par deux facteurs concomitants:

- La nature sémantique des caractéristiques. On a besoin d'une classification des caractéristiques telle que deux caractéristiques appartenant à une même classe sont homogènes et celles qui appartiennent à deux classes différentes, hétérogènes; c'est l'homogénéité/l'hétérogénéité sémantique. Par exemple, 'fatigué' et 'affamé' sont des caractéristiques sémantiquement homogènes; 'fatiguée' et 'belle' sont des caractéristiques sémantiquement hétérogènes.

- Un lien pragmatique entre les caractéristiques. Il s'agit d'un lien que le locuteur perçoit dans une situation d'énonciation donnée et qu'il veut exprimer; c'est l'homogénéité pragmatique. Ainsi, 'minuscule' et 'adorable' sont des caractéristiques sémantiquement hétérogènes; mais, dans une petite annonce, on parlera d'un chaton minuscule et adorable, car un petit être implique normalement l'attendrissement, ce que le locuteur tient à souligner. (On voit qu'en principe le facteur pragmatique l'emporte sur le facteur sémantique.)

Cette distinction a été signalée par Peškovskij (1934:391-392) et formulée explicitement par Boguslavskij (1996: 393), qui oppose l'homogénéité sémantique, ou intégrée (induite par le sens lexicographique des adjectifs), et l'homogénéité contextuelle, imposée par le locuteur cherchant à indiquer la similitude des caractéristiques d'un point de vue particulier.

Nous pouvons maintenant formuler des règles générales, plutôt banales, pour la correction des configurations sémantiques $\mathbf{A}$ et $\mathbf{B}$ :

- la configuration A est correcte si et seulement si ' $\sigma_{1}$ ' et ' $\sigma_{2}$ ' sont sémantiquement et pragmatiquement hétérogènes;

- la configuration B est correcte si et seulement si ' $\sigma_{1}^{\prime}$ ' et ' $\sigma_{2}$ ' sont soit (i) sémantiquement homogènes, soit (ii) sémantiquement hétérogènes, mais pragmatiquement homogènes.

Les configurations sémantiques A et B sont respectivement implémentées, dans la Structure Syntaxique Profonde, par les constructions «la subordination en parallèle» ( $\approx$ la codépendance) et «la coordination» (voir les constructions 1 et 2 ci-dessus, présentées dans le tableau 1). (La coordination, à son tour, peut utiliser une conjonction ou être asyndétique, c'est-à-dire n'être marquée que par la prosodie. Dans cet article, nous ne considérerons que la coordination par la conjonction ET / I.) Le choix entre les configurations $\mathbf{A}$ et $\mathbf{B}$ et, par ricochet, entre la codépendance et la coordination se réduit, comme on le voit, à la notion d'homogénéité des caractéristiques ' $\sigma_{1}$ ' et ' $\sigma_{2}$ ' ou des adjectifs correspondants $\mathrm{Adj}_{1}$ et $\mathrm{Adj}_{2}$. L'homogénéité sémantique peut être spécifiée par une classification sémantique des adjectifs; quant à l'homogénéité pragmatique, nous ne sommes pas en mesure de la modéliser formellement, puisque cela présuppose une représentation formelle de la réalité et de toutes nos connaissances. Ici, nous nous concentrons sur l'homogénéité sémantique des adjectifs. Comme le montrent les exemples en (7), cette homogénéité est relative à la langue; la section suivante discute donc des conditions d'homogénéité en russe et en français. 


\section{Homogénéité des adjectifs modificateurs}

\subsection{Adjectifs (quasi-)actantiels dans les deux langues}

Nous traiterons d'abord d'une famille d'adjectifs qui possèdent, en russe et en français, des propriétés communes du point de vue de leur coordonnabilité. Nous parlerons d'adjectifs relationnels $\left[=\mathrm{A}_{0}(\mathrm{~N})\right]$ qui expriment un actant sémantique du nom modifié, l'appartenance de ce nom (au sens le plus large possible) ou sa provenance; nous appellerons les adjectifs de la première classe actantiels (aide américaine) et ceux des deux dernières classes, quasi-actantiels (Petina kniga 'le livre de Petya'; voiture japonaise). Ce n'est qu'à l'intérieur de chaque classe ci-dessus que ces adjectifs sont homogènes et doivent être coordonnés; un adjectif (quasi-)actantiel ne peut donc pas être coordonné avec un adjectif de n'importe quelle autre classe (auxquels cas on utilise la codépendance):

(9) a. *l'aide américaine et immédiate *un attentat terroriste et abominable *l'amour maternel et fou

b. * ${ }^{*}$ staraja i Petina kniga 'vieux et de.Petya livre'

c. *une voiture japonaise et rouge brique *un roman russe et intéressant

Pour la construction où les deux adjectifs sont actantiels, il faut distinguer deux cas. Si ces adjectifs renvoient à deux actants différents du nom modifié, on ne peut pas les coordonner : ${ }^{*}$ aide américaine $_{\mathrm{X}}$ et alimentaire ( $_{\mathrm{Z}} \mathrm{X}$ aide $\mathrm{Y}$ par Z'); s'ils correspondent

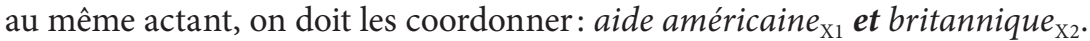

La particularité pertinente des adjectifs (quasi-)actanciels consiste en leur immunité absolue par rapport au facteur pragmatique. Ainsi, on ne peut pas dire ${ }^{\star}$ l'aide américaine et généreuse, même si le locuteur voit un lien logique entre les deux adjectifs (il pense que l'aide américaine est d'habitude généreuse).

Tous les autres adjectifs - ceux qui ne sont pas (quasi-)actantiels - doivent être distribués en des classes sémantiques de façon à ce que l'appartenance de deux adjectifs à une même classe signifie leur homogénéité (ce qui implique la possibilité de coordination). Une telle classification est spécifique de chaque langue.

\subsection{Homogénéité sémantique des adjectifs en russe}

Commençons par quelques exemples.

(10) *krasivye i čërnye glaza
'beaux et noirs yeux' $\quad \begin{aligned} & \text { krasivye čërnye glaza } \\ & \text { 'beaux noirs yeux' }\end{aligned}$

La couleur et l'évaluation esthétique sont trop éloignées (en russe!), c’est-à-dire non homogènes, pour qu'on les coordonne.

(11) umnyj i dobryj čelovek , $\quad$ *umnyj dobryj čelovek 'intelligent et bon homme' 'intelligent bon homme'

L'intelligence et la bonté sont trop homogènes - ce sont les qualités importantes de la personnalité - pour qu’on ne les coordonne pas. 
(12) a. Ego raspuxšie krasnye pal'cy bystro dvigalis' po klavišam.

'ses doigts rouges gonflés parcouraient vite le clavier'

b. Ivan s užasom smotrel na svoi raspuxšie i krasnye pal'cy.

'Ivan regardait, terrifié, ses doigts rouges et gonflés'

La couleur et l'état des doigts sont des caractéristiques assez hétérogènes, ce qui permet (12)a. Pourtant le locuteur peut les considérer comme assez homogènes, les deux décrivant un aspect (visuel) causant la terreur; (12)b est donc aussi possible.

L'homogénéité sémantique des caractéristiques est décrite pour le russe par la classification hiérarchique présentée dans le tableau 3 (d'après Iordanskaja 2000: 383-384; 2003: 161-162).

TABLEAU 3

\section{Classification sémantique des adjectifs en russe}

1. Caractéristiques objectives (non axiologiques et non attitudinales)

1.1. Caractéristiques qualificatives

1.1.1. Caractéristiques inhérentes

1.1.1.1. Caractéristiques inhérentes physiques

a. Matériau (kirpičnyj dom 'maison de.briques')

b. Texture (šeršavaja koža 'peau rugueuse')

c. Couleur (krasnaja mašina 'voiture rouge')

d. Forme (krugloe lico 'visage rond')

e. Autre (tjažëlaja kosa 'tresse lourde', udobnoe kreslo 'fauteuil confortable')

1.1.1.2. Caractéristiques inhérentes psychiques (genial'nyj filosof 'philosophe génial', glupyj čelovek 'homme stupide', umnoe lico 'visage intelligent')

1.1.1.3. Caractéristiques inhérentes abstraites

a. Espèce [au sens taxinomique] (dikoe / domašnee životnoe 'animal sauvage / domestique', rastitel'noe / slivočnoe maslo 'huile/ beurre')

b. Domaine (lingvističeskaja stat' ja 'article linguistique')

c. Intensité (glubokaja radost' 'joie profonde')

d. Autre (dorogaja mašina 'voiture chère')

1.1.2. Caractéristiques non inhérentes

1.1.2.1. Caractéristiques non inhérentes physiques

a. Localisation dans l'espace (sosednij dom 'maison voisine')

b. Localisation dans le temps (prošlogodnjaja vstreča 'rencontre de l'année dernière')

c. Autre (le plus souvent, état: ustalyj rabočij 'ouvrier fatigué', rvanye štany 'pantalon déchiré')

1.1.2.2. Caractéristiques non inhérentes psychiques (vljublënnyj filosof 'philosophe amoureux'; radostnyj golos 'voix joyeuse')

1.1.2.3. Caractéristiques non inhérentes abstraites (modnaja mašina 'voiture en vogue', estestvennoe ob"edinenie 'union naturelle')

1.2. Caractéristiques quantitatives (bol'šoj dom 'grande maison')

2. Caractéristiques subjectives

2.1. Caractéristiques axiologiques (zamečatel'nye glaza 'yeux magnifiques', potrjasajuščaja stat' ja 'article époustouflant', cennoe svojstvo 'propriété précieuse')

2.2. Caractéristiques attitudinales (ljubimaja kniga 'livre favori', dorogaja mama 'chère maman') 
Les caractéristiques ' $\sigma_{1}$ ' et ' $\sigma_{2}$ ', ainsi que les adjectifs correspondants $\operatorname{Adj}_{1}$ et $\operatorname{Adj}_{2}$, sont suffisamment homogènes si et seulement si elles se trouvent dans une même classe terminale de la hiérarchie; si ces caractéristiques sont dans des classes différentes, elles sont hétérogènes. (Rappelons que le locuteur est capable, dans certaines situations données, de leur trouver un aspect commun et, par là, de les rendre homogènes.)

Cette classification a été développée - suivant Vendler (1968) - dans le but de contrôler l'ordonnancement linéaire des adjectifs codépendants en russe:

$$
\begin{aligned}
& \text { bol'šoj kirpičnyj dom } \\
& \text { 'grande de.briques maison' }
\end{aligned}
$$

ou

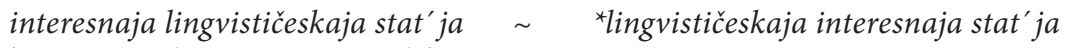
'intéressant linguistique article'

Mais cette même classification peut être utilisée, comme le prévoyait Vendler (1968: 123-125), pour spécifier les contraintes sur la coordination des adjectifs.

Le tableau 3 ne décrit que l'homogénéité sémantique des adjectifs; il fait abstraction des facteurs pragmatiques. (Par conséquent, certains de nos exemples illustrant le tableau 3 et marqués par un astérisque $\left(^{*}\right)$ peuvent être acceptables sous certaines conditions pragmatiques.) La classification présentée est, de façon évidente, liée aux composantes génériques dans les définitions des adjectifs (Iordanskaja 2003: 167), c'est-à-dire qu'elle est liée à leurs étiquettes sémantiques.

La classe terminale Autre est une division par défaut. Dans la hiérarchie, elle apparaît trois fois, lorsque la langue ne détermine pas franchement la capacité de coordonnabilité des adjectifs. C'est une espèce de fourre-tout, et des recherches approfondies peuvent mener à l'isolement de classes plus fines.

Illustrons le tableau 3 par des exemples.

a) Les adjectifs $\mathrm{Adj}_{1}$ et $\mathrm{Adj}_{2}$ sont hétérogènes, car ils se trouvent dans des classes terminales différentes, et n'affichent pas de lien pragmatique. Dans ce cas, la coordination des adjectifs est impossible et la codépendance s'impose.

- Caractéristique objective + caractéristique subjective

*potrjasajuščaja [car. axiologique, 2.1.] i naučnaja [car. objective: domaine, 1.1.1.3.b.] stat' ja

'article époustouflant et scientifique'

$<$ potrjasajuščaja naučnaja stat' ja>

*prijatnaja [2.1.] i prošlogodnjaja [1.1.2.1.b.] vstreča

'rencontre plaisante et d'année.dernière'

$<$ prijatnaja prošlogodnjaja vstreča $>$

*blistatel'noe [2.1.] i kul'turnoe [1.1.1.3.b.] prošloe

'passé brillant et culturel'

<blistatel'noe kul'turnoe prošloe>

- Caractéristique qualificative + caractéristique quantitative

*bol'šoj [car. quantitative, 1.2.] i kirpičnyj [car. qualificative: matériau, 1.1.1.1.a.] dom 'maison grande et de.briques'

$<$ bol'šoj kirpičnyj dom> 
*bol'šaja [1.2.] i krasnaja [1.1.1.1.c.] roza

'rose grande et rouge'

$<$ bol'šaja krasnaja roza>

- Caractéristique inhérente + caractéristique non inhérente

*genial'nyj [car. inhérente psychique, 1.1.1.2.] i vljublënnyj [car. non inhérente psychique, 1.1.2.2.] filosof

'un philosophe génial et amoureux'

$<$ vljublënnyj genial'nyj filosof>

*ispugannye [1.1.2.2.] i čërnye [1.1.1.1.c.] glaza

'yeux effrayés et noirs'

<ispugannye čërnye glaza>

- Caractéristique psychique + caractéristique physique

*umnoe [car. inhérente psychique, 1.1.1.2.] i krugloe [car. inhérente physique, 1.1.1.1.d.]

lico

'visage rond et intelligent'

$<$ umnoe krugloe lico>

${ }^{*}$ zagoreloe [car. non inhér. physique, 1.1.2.1.c.] i radostnoe [car. non inhér. psychique, 1.1.2.2.] lico

'visage bronzé et joyeux'

$<$ zagoreloe radostnoe lico $>$

- Deux caractéristiques qui ne diffèrent qu'au niveau terminal

*blestjaščie [1.1.1.1.e.] i čërnye [1.1.1.1.c.] glaza

'yeux brillants et noirs'

$<$ blestjaščie čërnye glaza>

b) Les adjectifs $\mathrm{Adj}_{1}$ et $\mathrm{Adj}_{2}$ sont homogènes, car ils sont dans une même classe terminale qui n'est pas Autre - sauf «état»; la coordination est indispensable, et la codépendance est impossible:

umnyj [car. inhérente psychique, 1.1.1.2.] $\boldsymbol{i}$ dobryj [1.1.1.2.] čelovek

'homme intelligent et bon'

$<^{*}$ umnyj dobryj čelovek>

ustalyj [car. non inhérente physique: état, 1.1.2.1.c.] i golodnyj [1.1.2.1.c.] rebënok 'enfant fatigué et affamé'

$<^{*}$ ustalyj golodnyj rebënok>

Dorogaja [car. attitudinale, 2.2.] i glubokouvažaemaja [2.2.] Anna Andreevna!

'Chère et estimée A. A.'

$<{ }^{*}$ Dorogaja glubokouvažaemaja Anna Andreevna!>

estestvennoe [car. non inhérente abstraite, 1.1.2.3.] i dobrovol'noe [1.1.2.3.] ob"edinenie narodov

'union des peuples naturelle et volontaire'

$<^{*}$ estestvennoe dobrovol'noe ob"edinenie narodov>

Remarquons que l'exclusion de caractéristiques appartenant à la classe Autre de ce cas de figure (la coordination est indispensable) est due au fait que cette classe 
réunit des caractéristiques qui ne se prêtent pas à une taxinomie bien claire. Les classes Autre sont une source de coordination optionnelle, comme le montre le cas suivant.

c) Les adjectifs $\mathrm{Adj}_{1}$ et $\mathrm{Adj}_{2}$ sont:

(i) soit homogènes, bien qu'à un degré faible, s'ils se trouvent dans une même classe Autre;

(ii) soit hétérogènes, s'ils se trouvent dans des classes terminales différentes, mais sont tels que le locuteur y voit un fondement commun (donc, s'ils sont pragmatiquement homogènes).

Dans ce cas de figure, la coordination et la codépendance sont toutes les deux permises:

Le cas (i)

xolodnyj [car. obj.: autre, 1.1.1.1.e.] i tixij $\quad \sim \quad$ xolodnyj tixij večer

[car. obj.: autre, 1.1.1.1.e.] večer

'soirée fraîche et calme'

skol'zkie [1.1.2.1.c.] i mokrye [1.1.2.1.c.] ～～skol'zkie mokrye stupeni

stupeni

'marches glissantes et mouillées'

Cependant, à cause de la nature vague des classes Autre, certaines caractéristiques s'y trouvant ne peuvent pas être coordonnées: ${ }^{\star}$ tonkaja [1.1.1.1.e.] i vyšitaja [1.1.1.1.e.] bluzka 'blouson mince et brodé'. Pour couvrir des cas comme celui-ci, il faut une classification plus fine. D'autre part, dans de tels cas, on pourrait avoir recours aux composantes génériques des adjectifs en question; ainsi, pour TONKIJ 'dont l'épaisseur est réduite' et VYšiTYj 'décoré de broderies', on voit qu'ils sont hétérogènes.

Le cas (ii)

gromkij [1.1.1.1.e.] $\boldsymbol{i}$ vlastnyj [1.1.1.2.] golos $\sim$ gromkij vlastnyj golos

'voix forte et autoritaire'

bol'šoe [1.2.] i udobnoe [1.1.1.1.e.] kreslo $\sim$ bol'šoe udobnoe kreslo

'fauteuil grand et confortable'

On voit que le locuteur peut violer la classification sémantique, quand il veut rapprocher les caractéristiques dont il s'agit; il le fait en coordonnant par I 'et' les adjectifs qui ne sont pas sémantiquement homogènes selon le tableau 3. Ce n'est possible que quand le locuteur perçoit, dans une situation donnée, un aspect qui réunit les deux caractéristiques hétérogènes et veut l'exprimer. Par exemple, en (13)a, selon le tableau 3, la conjonction ne doit pas apparaître (car 'étroit' est une caractéristique quantitative mais 'à pic' est qualitative); pourtant, en (13)b, elle est acceptable sans problème, car le locuteur veut insister sur les deux propriétés du toit qui ensemble rendent la descente difficile:

(13) a. Na fotografii xorošo vidna uzkaja

??Na fotografii xorošo vidna

krutaja kryša ètogo doma. uzkaja i krutaja kryša ètogo doma.

'Sur la photo on voit bien un toit étroit à.pic de cette maison’ 
b. Ivan lovko spustilsja po uzkoj i krutoj kryše.

'Ivan a adroitement descendu le toit étroit et à.pic'

En (14)b, le locuteur souligne un aspect commun de 'jeune' et de 'beau' dans ce contexte particulier: les deux propriétés constituent un atout pour être engagé.

(14) a. V komnatu vošla krasivaja molodaja ženščina.

'Il est entré dans la pièce une jeune belle femme'
?? V komnatu vošla krasivaja $\boldsymbol{i}$ molodaja ženščina.

b. Krasivaja i molodaja ženščina naidët rabotu bystree, čem nekrasivaja i staraja. 'Une belle et jeune femme trouvera du travail plus vite qu'[une] laide et vieille'.

Rappelons également un bel et frappant exemple de Peškovskij (1934: 392), qui insiste sur le point de vue du locuteur dans une situation donnée: une entremetteuse, en louant la dot de sa protégée, dit que la jeune fille possède bol'šoj [1.2] i kamennyj [1.1.1.1a] dom ('maison grande et en.pierre'), cette coordination étant impossible hors d'un contexte très spécial (une mise en valeur de la maison).

\subsection{Homogénéité sémantique des adjectifs en français}

Selon les exemples en (7), le français emploie, dans de nombreux cas, des adjectifs coordonnés par ET, alors qu'en russe, la coordination des adjectifs correspondants par I est impossible et que la construction avec les adjectifs codépendants prévaut. Cette divergence est due à la permissivité plus grande du français vis-à-vis du degré d'homogénéité sémantique des adjectifs coordonnés. (De même sur le plan syntaxique: plus permissif, le français coordonne les adjectifs avec les relatives, ce qui est exclu en russe.) Il s'ensuit que la classification sémantique des adjectifs français peut être différente de ce qui est proposé dans le tableau 3. Nous ne sommes pas en mesure d'élaborer une telle classification de façon sérieuse; néanmoins, une enquête rapide parmi des informateurs francophones et des recherches sur l'Internet nous permettent de formuler quelques observations utiles. Ce faisant, nous allons nous référer au tableau 3, même si nous avons introduit une nouvelle opposition pertinente pour le français: [caractéristique] visuelle non visuelle (une caractéristique visuelle est directement perçue par la vue).

Par rapport à la coordonnabilité des adjectifs, le français distingue les mêmes trois cas de figure que le russe.

a) Les adjectifs $\mathrm{Adj}_{1}$ et $\mathrm{Adj}_{2}$ sont hétérogènes et le locuteur ne leur trouve pas un fondement pragmatique commun : leur coordination est impossible et la codépendance s'impose.

L'implication est la même qu'en russe, sauf que l'éloignement des classes sémantiques est compris en français de façon beaucoup plus libérale. Tandis que le russe considère comme hétérogènes des caractéristiques appartenant à des classes terminales différentes du tableau 3, le français traite comme sémantiquement assez homogènes - et donc coordonnables par ET - un nombre élevé de paires de telles caractéristiques. Les adjectifs non coordonnables en français peuvent être spécifiés par quelques contraintes générales. 
Soit deux caractéristiques pragmatiquement non liées (autrement dit, la première n'entraînant pas la seconde et les deux ne correspondant pas à une même valeur axiologique). Le français ne permet pas de coordonner par ET ces deux caractéristiques dans les conditions suivantes:

1) Si une caractéristique est objective et l'autre subjective:

*un article scientifique et intéressant

${ }^{*}$ un passé culturel et brillant

${ }^{*}$ une rencontre annuelle et plaisante

$\sim \quad$ un article scientifique intéressant
$\sim \quad$ un passé culturel brillant
$\sim \quad$ une rencontre annuelle plaisante

Cependant, si le locuteur voit un lien pragmatique entre la caractéristique objective et l'évaluation subjective, la conjonction ET est possible:

un minuscule et magnifique chiot (un petit être normalement implique l'attendrissement)

un service long et piteux (les deux caractéristiques sont fortement négatives)

une punition atroce et disproportionnée (les deux caractéristiques sont évidemment négatives)

2) Si les caractéristiques appartiennent toutes les deux à des catégories sémantiques bien définies, telles les dimensions, la forme, la couleur, la fréquence, la localisation, etc. :

*une vallée triangulaire et profonde

${ }^{*}$ une église blanche et miniature

${ }^{*}$ un bulletin mensuel et international une vallée triangulaire profonde

$\sim \quad$ une église blanche miniature

$\sim \quad$ un bulletin mensuel international

3) Si une caractéristique est visuelle et l'autre, non visuelle:

$$
\begin{aligned}
& \text { *une voiture rouge et fiable } \sim \text { une voiture rouge fiable } \\
& { }^{*} \text { une voiture rouge et très chère } \sim \text { une voiture rouge très chère } \\
& \text { *une fille blonde et intelligente } \sim \text { une fille blonde intelligente }
\end{aligned}
$$

Remarquons que les expressions correctes du type une fille belle et intelligente ou une voiture grande et pas chère (le premier adjectif est visuel et le second non) ne contredisent pas notre affirmation. Il s'agit en effet de la coordination de deux caractéristiques pragmatiquement homogènes - dans les deux expressions, les propriétés en question sont positives, de sorte que ces expressions sont prévues par le cas c-(ii) p. 182.

4) Si une caractéristique est visuelle inhérente (permanente) et l'autre, visuelle non inhérente (temporaire) $)^{7}$ :

*un sommet conique et enneigé

*une vallée profonde et inondée $\sim \quad$ un sommet conique enneigé

une vallée profonde inondée

À noter que si elles ne sont pas visuelles, les caractéristiques permanente et temporaire sont coordonnables, comme dans un philosophe génial et amoureux.

Toutes les paires d'adjectifs qui ne sont pas explicitement interdites sont admises. Ainsi, par exemple, deux caractéristiques objectives inhérentes qui appartiennent à deux classes différentes, mais ne représentent pas des catégories sémantiques bien définies peuvent être coordonnées - pourvu que les deux soient visuelles (un sentier 
étroit et sablonneux, un visage rond et intelligent, une peau luisante et tendue, des quenottes luisantes et microscopiques, un calot rigide et brodé, ...).

b) Les adjectifs $\mathrm{Adj}_{1}$ et $\mathrm{Adj}_{2}$ sont homogènes, car ils sont:

(i) soit dans une même classe terminale qui n'est pas Autre;

(ii) soit l'un des deux se trouve dans une classe Autre, les deux exprimant des propriétés visuelles.

Dans ce cas, la coordination est indispensable, et la codépendance impossible:

Le cas (i)

un homme bon [car. inhérente psychique, ${ }^{*}$ un homme bon intelligent

1.1.1.2.] et intelligent [car. inhérente

psychique, 1.1.1.2.]

un enfant affamé [car. non inhérente physique: état, 1.1.2.1.c.] et fatigué [car.

*un enfant affamé fatigué non inhérente physique: état, 1.1.2.1.c.]

cher [car. attitudinale, 2.2.] et estimé [car. $\quad{ }^{*}$ Cher estimé collègue! attitudinale, 2.2.] collègue!

Le cas (ii)

La maison rouge [1.1.1.1.c.] et spacieuse $\quad \sim \quad{ }^{\star}$ La maison rouge spacieuse, [1.1.1.1.e.], dont il avait hérité... dont il avait hérité...

c) Les adjectifs $\mathrm{Adj}_{1}$ et $\mathrm{Adj}_{2}$ sont:

(i) soit homogènes, s'ils se trouvent dans une même classe Autre;

(ii) soit hétérogènes, s'ils se trouvent dans des classes terminales différentes, mais sont tels que le locuteur y voit un fondement commun (donc s'ils sont pragmatiquement homogènes).

Dans ce cas de figure, la coordination et la codépendance sont toutes les deux permises. Cependant, la coordination est de loin préférée: pour nos exemples, d'après nos informateurs, la version avec coordination serait la meilleure. Quant à la codépendance, nous avons trouvé certains désaccords entre informateurs, que nous n'avons pas marqués ici et qui sont dus aux perceptions pragmatiques différentes des relations entre les deux caractéristiques (plus hétérogènes pour un locuteur que pour un autre):

Le cas (i)

cet après-midi doux [1.1.2.1.c.] et ensoleillé [1.1.2.1.c.]

un blouson mince [1.1.1.1.e.] et brodé [1.1.1.1.e.]

des marches mouillées [1.1.2.1.c.] et glissantes [1.1.2.1.c.]

Le cas (ii) une voix basse [1.1.1.1.e.] et séduisante [1.1.1.2.] [une voix basse (féminine) implique la séduction]

～cet après-midi doux ensoleillé

un blouson mince brodé

$\sim$ des marches mouillées glissantes

- une voix basse séduisante 
une punition cruelle [2.1.] et arbitraire [1.1.1.3.d.]

[une punition très mauvaise]

une salle chaude [1.1.1.1.e.] et énorme [1.2.]

[une salle confortable]

un isthme étroit [1.2.] et sablonneux

[1.1.1.1.e.]

[les deux caractéristiques sont visuelles]

un visage rond [1.1.1.1.d.] et intelligent

[1.1.1.2.]

[les deux caractéristiques sont visuelles]

une musique moderne [1.1.1.3.d.] et douce une musique moderne douce

[1.1.2.1.c.]

[les deux caractéristiques sont positives

pour le locuteur]

Cependant, la coordination peut être indispensable (et la codépendance impossible ou, du moins, non souhaitable) si les deux caractéristiques possèdent un lien pragmatique très fort:

\begin{tabular}{|c|c|c|}
\hline $\begin{array}{l}\text { sa peau tendre [1.1.1.1.e.] et chaude } \\
\text { [1.1.1.1.e.] }\end{array}$ & $\sim$ & ${ }^{*}$ sa peau tendre chaude \\
\hline $\begin{array}{l}\text { une pièce chaude [1.1.1.1.e.] et lumineuse } \\
\text { [1.1.1.1.e.] }\end{array}$ & $\sim$ & *une pièce chaude lumineuse \\
\hline $\begin{array}{l}\text { son visage bronzé [1.1.2.1.c.] et joyeux } \\
{[1.1 .2 .2 .]}\end{array}$ & $\sim$ & 'son visage bronzé joyeux \\
\hline $\begin{array}{l}\text { son visage bête [1.1.1.2.] et bouffi } \\
\text { [1.1.1.1.e.] }\end{array}$ & $\sim$ & 'son visage bête bouffi \\
\hline un roman long [1.2.] et dégoûtant [2.1.] & $\sim$ & 'un roman long dégoûtant \\
\hline
\end{tabular}

\subsection{Coordination vs codépendance des adjectifs modificateurs en russe et en français: une comparaison}

De façon générale, le français rapproche et traite comme coordonnables beaucoup plus de paires de caractéristiques adjectivales que le russe. Dans ce qui suit, nous allons insister sur les différences majeures que nous avons été en mesure d'établir.

- Peuvent être coordonnées en français, mais pas en russe (où l'on voit la codépendance), les caractéristiques de types suivants:

a) Une caractéristique objective et une caractéristique subjective si les deux sont pragmatiquement assez rapprochées:

la petite et adorable Kim un chaton tout noir et adorable un roman long et dégoûtant

$\quad{ }^{*}$ malen'kaja i očarovatel'naja Kim

〜 ${ }^{*}$ sovsem čërnyj i očarovatel'nyj kotënok

$\sim \quad{ }^{*}$ dlinnyj i otvratitel'nyj roman

Le français donne un rôle plus crucial au facteur pragmatique; le russe s'appuie plutôt sur l'homogénéité sémantique des caractéristiques, de sorte qu'une coordina- 
tion de ce type est possible (par exemple, dlinnyj i skučnyj roman 'un roman long et ennuyeux') mais plus restreinte.

b) Deux caractéristiques visuelles, même si elles appartiennent à des classes sémantiques différentes:

des yeux noirs et brillants

un isthme étroit et sablonneux

une maison rouge et spacieuse

un blouson mince et brodé

*blestjaščie i čërnye glaza

$\quad{ }^{*} u z k i j$ i pesčanyj perešeek

$\sim \quad{ }^{*}$ krasnyj i prostornyj dom

${ }^{*}$ tonkaja i vyšitaja bluzka

Le français attribue une beaucoup plus grande importance à la dimension de visualité.

c) Deux caractéristiques non visuelles si l'une est physique et l'autre, abstraite:

un petit pain chaud et pas cher $\quad \sim$ ?dešëvaja i gorjačaja buločka

une musique moderne et douce $\quad \sim \quad{ }^{*}$ sovremennaja i tixaja muzyka

d) Deux caractéristiques psychiques, même si l'une est permanente et l'autre, temporaire:

un philosophe génial et amoureux

un enfant intelligent et surexcité
* *vljublënnyj i genial'nyj filosof

*umnyj i perevozbuždënnyj rebënok

- Peuvent être codépendantes en russe, mais pas en français (qui impose la coordination), des caractéristiques visuelles appartenant à des classes sémantiques différentes mais non pas à deux catégories bien définies (voir 3.3., a-2, p. 180):
glupoe odutlovatoe lico
$\sim \quad *$ un visage bête bouffi
vysokoe sinee nebo
$\sim \quad{ }^{*}$ un ciel haut bleu

Cependant, la maison rouge spacieuse est correct, puisque 'rouge' et 'spacieux' appartiennent à des catégories bien définies (la couleur et la grandeur).

En résumé, le français est plus libéral avec la coordination des adjectifs modificateurs, alors que le russe l'est avec leur codépendance. Comme conséquence, dans les cas optionnels, le français préfère la coordination. En même temps, les deux langues manifestent bien des parallélismes, que nous illustrerons par quelques exemples:

a) La coordination est impossible en français comme en russe si une des caractéristiques est objective et l'autre, subjective, et s'il n'y a pas de lien pragmatique entre les deux (dans ce cas, on utilise la codépendance):

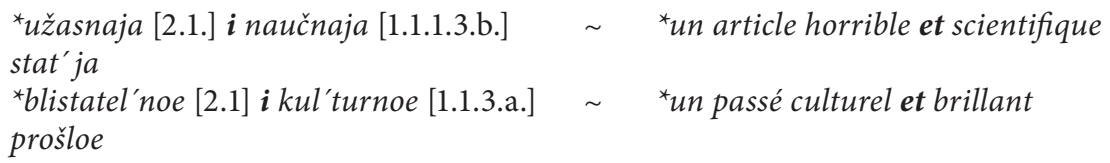

b) La coordination est indispensable quand les deux caractéristiques se trouvent dans une même classe terminale qui n'est pas Autre, sauf «état» (la codépendance est exclue):

umnyj [1.1.1.2.] i dobryj [1.1.1.2.] čelovek $\sim$ un homme bon et intelligent ustalyj [1.1.2.1.c.] i golodnyj [1.1.2.1.c.] un enfant affamé et fatigué rebënok 
c) La coordination est optionnelle quand les deux caractéristiques se trouvent dans des classes différentes et qu'il existe un lien pragmatique entre les deux:

žestokoe [2.1.] (i) nezaslužennoe [1.1.1.3.d.] nakazanie

ogromnyj [1.2.] (i) tëplyj [1.1.1.1.e.] dom une punition cruelle (et) arbitraire une maison chaude (et) énorme

Outre les contraintes sémantiques sur l'homogénéité des adjectifs en français et en russe, il serait nécessaire de considérer l'aspect syntaxique de la coordination des adjectifs modificateurs. Nous ne pouvons malheureusement pas le faire et nous nous limiterons à quelques remarques. Le français tolère moins bien que le russe la codépendance des deux adjectifs modificateurs qui se suivent. Si un des adjectifs permet l'antéposition, on préfère le mettre devant le nom et laisser l'autre derrière: une jolie tête maladive, une grande salle chaude, une profonde vallée triangulaire, etc. Sinon, on peut encore introduire la coordination asyndétique: sa peau luisante, tendue; chaussée mouillée, glissante; etc. Ce facteur syntaxique brouille les cartes, en diminuant le nombre de cas où le locuteur a vraiment le choix libre entre la coordination et la codépendance des adjectifs en postposition. En fait, le problème de coordonnabilité des adjectifs est lié au problème de leur ordonnancement, que nous avons soigneusement évité dans cet article.

\section{Conclusion}

En guise de conclusion, nous aimerions faire trois remarques de caractère général. Premièrement, le cadre logique de la présente étude est en fait l'établissement des contraintes sur la correction linguistique des Structures Sémantiques, problème soulevé dans Iordanskaja et Mel'čuk (2009). Le présent article fait état d'un nouvel exemple, assez complexe, non évident, de différences entre les langues se situant sur le plan sémantique. D’une part, les contraintes discutées s'attachent, en partie, au sémantème 'ensemble ${ }_{\mathrm{et}}$ ' (c'est-à-dire à une acception de la conjonction I / ET) et, en partie, à une configuration particulière de sémantèmes: notre configuration $\mathbf{A}$ (tableau 2), qui détermine la codépendance; cela est un nouveau type formel de contrainte. D'autre part, dans la formulation des contraintes sur la coordination, des considérations pragmatiques (impliquant le locuteur et la situation d'énonciation) jouent un rôle, ce qui n'est pas le cas pour les contraintes que nous avons formulées auparavant.

Deuxièmement, notre recherche confirme la thèse d'Apresjan (1989 [1995: 624625]): si l'expression linguistique d'une anomalie sémantique (au sens le plus large) n'est pas assez explicite, cela mène à une anomalie linguistique. Dans notre cas, les contraintes proposées ne sont valides que pour des adjectifs en tant que modificateurs. Si deux caractéristiques, hétérogènes d'après notre classification, s'expriment comme attributs d'une copule - en particulier, par des relatives -, ces combinaisons sont linguistiquement normales, tout en restant peut-être pragmatiquement bizarres:

\section{(15) *cette voiture rouge et très chère}

cette voiture était rouge et très chère cette voiture, qui était rouge et très chère

Nous considérons l'attribution des propriétés au moyen d'une copule comme étant plus explicite que celle au moyen de la modification adjectivale (car la première est plus proche de la structure sémantique). 
Troisièmement, en réalité, le choix entre coordination et codépendance est également affecté, dans les deux langues, par un facteur de nature communicative. Si le locuteur veut parler d'une jolie femme qui est jeune, ce qui présuppose un bloc sémantique 'une jolie femme', la coordination est impossible, et la codépendance s'impose; mais s'il vise le sens 'une femme' et veut associer à la femme deux caractéristiques, la coordination est possible:

(16) a. 'une jolie femme qui est jeune' $\Leftrightarrow$ une jolie femme jeune [mais pas une jeune et jolie femme pour ce sens]

b. 'une femme qui est jolie et jeune' $\Leftrightarrow$ une jeune et jolie femme [mais pas une jolie femme jeune pour ce sens]

Une description plus fine et plus stricte devra prendre en considération ce facteur; mais cela, c'est un nouveau défi!

\section{REMERCIEMENTS}

Les premières ébauches de cet article ont été lues et critiquées par I. Boguslavskij, G. Gross, S. Kahane, J. Lévy et E. Savvina; le texte final a subi l'inspection sévère, mais juste, de S. Vandaele. Nous tenons à exprimer notre reconnaissance la plus profonde à ces collègues et amis. Les erreurs et défauts qui ont survécu à leur lecture ne sont imputables qu'à nous-mêmes.

\section{NOTES}

* Observatoire de linguistique Sens-Texte.

1. Giroud, Françoise (2002): Lou, histoire d'une femme libre. Paris: Fayard.

2. Symboles utilisés dans l'article: ${ }^{*}$ : expression incorrecte; ' $:$ expression discutable/douteuse; $<>$ : variante; $\sim$ : 'est en relation avec', 'est opposé à'; $\Leftrightarrow$ : 'est exprimé par'; les points entre les gloses françaises indiquent que les gloses réunies correspondent à un seul mot en russe ('tout.le.monde' $\sim$ vse; 'de.briques' kirpičnyj).

3. La conjonction française ET est utilisée de façon beaucoup plus libre que l'I russe. Elle peut coordonner non seulement un adjectif avec une relative (par exemple, les esprits justes et qui aiment faire $d u$ bien), ce qui est complètement exclu en russe, mais aussi un syntagme prépositionnel avec une proposition circonstancielle ([c]'était dans le couloir où, pour le premier jour d'école et parce $q u$ 'il pleuvait, on avait entassé les enfants).

4. Les adjectifs coordonnés par la conjonction MAIS (français) / NO (russe) ne sont pas considérés dans cet article, car ils ne forment pas un ensemble conjonctif ('ensemble ${ }_{\mathrm{et}}$ '): les propriétés exprimées ne sont pas "égales» ('petit, mais fort' $\neq$ 'fort, mais petit', contrairement à 'petit et fort' = 'fort et petit').

5. Insistons sur le fait que, dans une construction quasi coordinative, l'emploi d'une conjonction est impossible.

6. Les sémantèmes ' $\sigma_{1}$ ' et ' $\sigma_{2}$ ' ne doivent pas être tous les deux communicativement dominants par rapport au nom modifié. Si c'est le cas, ils constituent nécessairement un ensemble conjonctif (= sont dépendants du sémantème 'ensemble ${ }_{\mathrm{et}}$ ') et donnent, dans la structure syntaxique, l'attribut coordonné d'une copule, et non pas des modificateurs du nom.

7. La distinction [propriété] permanente vs temporaire n'est pas toujours évidente. Ainsi, les syntagmes chemin étroit et boueux et chemin étroit boueux sont possibles tous les deux, car 'boueux' peut être considéré comme une propriété permanente ou temporaire.

\section{RÉFÉRENCES}

Apresjan, Jurij (1989/1995): Tavtologičeskie i kontradiktornye anomalii. In: Jurij ApresJan, dir. Izbrannye trudy. Tom II. Integral'noe opisanie jazyka i sistemnaja leksikografija, Moskva: Škola «Jazyki russkoj kul'tury», 622-628.

Boguslavskij, Igor (1996): Sfera dejstvija leksičeskix edinic. Moskva: Škola «Jazyki russkoj kul'tury». 
IORDANSKAJA, Lidija (2000): Sopodčinenie prilagatel'nyx v russkom jazyke. In: Leonid IomDIN et Leonid Krysin, dir. Slovo v tekste i v slovare, Moskva: Jazyki russkoj kul'tury, 379-390. IORDANSKAJA, Lidija (2003): L’ordonnancement des adjectifs codépendants en russe. In: Sylvain Kahane et Alexis NasR, dir. Proceedings of the First International Conference on MeaningText Theory (MTT'03). Paris: École normale supérieure, 159-169.

Iordanskaja, Lidija et MeL'Čuk, Igor (2009): Linguistic Well-Formedness of Semantic Structures. In: David Beck, Kim Gerdes, Jasmina Milićević, et al., dir. Proceedings of the Fourth International Conference on Meaning-Text Theory (MTT'09). Montréal, 177-187. Consulté le 29 janvier 2010, <http://meaningtext.net/mtt2009>.

PešKovskiJ, Aleksandr (1934): Russkij sintaksis v naučnom osvě̌čenii. Moskva: Učpedgiz.

SANNikov, Vladimir (1989): Russkie sočinitel'nye konstrukcii. Moskva: Nauka.

Vendler, Zeno (1968): Adjectives and Nominalizations. The Hague - Paris: Mouton.

Wierzbicka, Anna (1980): Lingua Mentalis. The Semantics of Natural Language. Sydney: Academic Press. 